\title{
ON A HYPERCYCLE SYSTEM WITH NONLINEAR RATE
}

\author{
JUNCHENG WEI* AND MATTHIAS WINTER ${ }^{\dagger}$
}

\begin{abstract}
We study a $(N+1)$-hypercyclical reaction-diffusion system with nonlinear rate $p$. It is shown that there exists a critical threshold $N_{0}$ such that for $N \leq N_{0}$ the system is stable while for $N>N_{0}$ it becomes unstable. It is also shown that for large $p, N_{0}$ remains a constant: in fact for $p \geq p_{0} \sim 4.35, N_{0}=5$ and for $p<p_{0} \sim 4.35, N_{0}=4$.
\end{abstract}

1. Introduction. In this paper, we are concerned with the following reactiondiffusion system:

(1.1) $\left\{\begin{array}{l}\frac{\partial X_{i}}{\partial t}=D_{X} \frac{\partial^{2} X_{i}}{\partial x^{2}}-g_{X} X_{i}+M \sum_{j=1}^{N} k_{i j} X_{i} X_{j}^{n}, \quad i=1,2, \ldots, N, \quad x \in R, \\ \frac{\partial M}{\partial t}=D_{M} \frac{\partial^{2} M}{\partial x^{2}}+k_{M}-g_{M} M-L M \sum_{i, j=1}^{N} k_{i j} X_{i} X_{j}^{n}, \quad x \in R,\end{array}\right.$

where $X_{i}$ denotes the concentration of the polymers, and $M$ is the concentration of activated monomers. $N$ is the number of different polymer species. The replication of each polymer $X_{i}$ is catalysed by each $X_{j}$ at a constant rate $k_{i j}$. Linear (noncatalytic) growth terms are neglected. The activated monomers are produced at a constant rate, $k_{M} ; g_{X}$ and $g_{M}$ are decay rate constants. $L$ is the number of monomers in each polymer, and $D_{X}$ and $D_{M}$ are constant diffusion coefficients. The exponent $n$ is a positive number.

We assume that the coefficients $k_{i j}$ are represented by a hypercyclical $N \times N$ matrix,

$$
\left(k_{i j}\right)=\left(\begin{array}{ccccc}
0 & 0 & \cdots & 0 & k_{0} \\
k_{0} & 0 & \cdots & 0 & 0 \\
0 & k_{0} & 0 & \cdots & 0 \\
0 & 0 & \cdots & \cdots & 0 \\
0 & 0 & \cdots & k_{0} & 0
\end{array}\right)_{N \times N}, \quad k_{0}>0
$$

When $n \neq 1$, We call (1.1) is a hypercycle system with nonlinear rate. The reason is the following: at each $X_{i}$, the kinetic reaction rate is given by

$$
\Gamma_{i}=-g_{X}+M \sum_{j=1}^{N} k_{i j} X_{j}^{n}
$$

When $n=1$, we have a linear growth rate for $\Gamma_{i}$ and the system is called classical hypercycle system. When $n<1$, the growth rate is sublinear and $n>1$ the growth rate is superlinear. Such nonlinear reaction rates were also introduced and studied in the one component case, i.e., $N=1$, by many authors, see [18], [19], [20] and the references therein.

The classical hypercycle system arises as a spatial model concerning the origin of life similar to the one introduced by Eigen and Schuster [12]. A number of RNAlike polymers ("components") catalyse the replication of each other in a cyclic way.

\footnotetext{
*Department of Mathematics, The Chinese University of Hong Kong, Shatin, Hong Kong (wei@math.cuhk.edu.hk).

†Mathematisches Institut, Universität Stuttgart, D-70511 Stuttgart, Germany (winter@mathematik.uni-stuttgart.de).
} 
Examples in nature include Krebs and Bethe-Weizsäcker cycles. Eigen and Schuster argue that the hypercycle satisfies important criteria of natural selection: 1. Selective stability of each component due to favorable competition with error copies, 2. Cooperative behavior of the components integrated into the hypercycle, and 3. Favorable competition of the hypercycle unit with other less efficient systems.

Nonlinear rates are interesting to model different coupling strength of the various components, where a higher rate $n$ corresponds to stronger coupling.

We consider stationary cluster-like solutions of (1.1). A cluster may loosely be defined as a region of high concentration $\sum_{i=1}^{N} X_{i}$ of the polymers and low concentration of the monomer, as monomers are consumed by the replication of polymers. (If the region shrinks to a point, this phenomenon is called point-condensation.)

Cluster solutions for (1.1) have been studied by numerous authors. For numerical aspects of clusters, see [3], [5], [6], [7] and the references therein. In [35], we first made a rigorous study on the existence and stability of cluster solutions of hypercycle system with linear rate $(n=1)$ in $R^{2}$ : it was shown that for $N \leq 4$, cluster solutions are stable while for large $N$, cluster solutions become unstable. In [35], we analyzed the cluster solutions in $R^{1}$ for $n=1$ and we found the exact threshold for $N$. More precise statements of the results of [35] will be stated later.

Let us first reduce the system (1.1) to standard form. Dividing by $g_{X}$ and $g_{M}$, respectively, gives

$$
\begin{gathered}
\frac{1}{g_{X}} \partial_{t} X_{i}=\frac{D_{X}}{g_{X}} X_{i}^{\prime \prime}-X_{i}+\frac{M}{g_{X}} \sum_{j=1}^{N} k_{i j} X_{i} X_{j}^{n}, x \in R \\
\frac{1}{g_{M}} \partial_{t} M=\frac{D_{M}}{g_{M}} M^{\prime \prime}+\frac{k_{M}}{g_{M}}-M-\frac{L M}{g_{M}} \sum_{j i,=1}^{N} k_{i j} X_{i} X_{j}^{n}, x \in R .
\end{gathered}
$$

Rescaling $M=\left(k_{M} / g_{M}\right) \hat{M}, X_{i}=\left(g_{M} / L\right)^{1 /(n+1)} \hat{X}_{i}$, we get

$$
\begin{gathered}
\frac{1}{g_{X}} \partial_{t} \hat{X}_{i}=\frac{D_{X}}{g_{X}} \hat{X}_{i}^{\prime \prime}-\hat{X}_{i}+\frac{1}{g_{X}} \frac{k_{M}}{g_{M}} \hat{M}\left(\frac{g_{M}}{L}\right)^{n /(n+1)} \sum_{j=1}^{N} k_{i j} \hat{X}_{i} \hat{X}_{j}^{n} \\
\frac{1}{g_{M}} \partial_{t} \hat{M}=\frac{D_{M}}{g_{M}} \hat{M}^{\prime \prime}+1-\hat{M}-\hat{M} \sum_{i, j=1}^{N} k_{i j} \hat{X}_{i} \hat{X}_{j}^{n} .
\end{gathered}
$$

Rescaling space variables $x$ and time variable $t$ :

$$
x=\sqrt{\frac{D_{M}}{g_{M}}} \hat{x}, \quad t=\frac{1}{g_{M}} \hat{t}
$$

renaming constants:

$$
A=\frac{k_{M}}{g_{X} g_{M}}\left(\frac{g_{M}}{L}\right)^{\frac{n}{n+1}}, \quad \epsilon^{2}=\frac{D_{X}}{D_{M}} \frac{g_{M}}{g_{X}}, \quad \tau=\frac{g_{X}}{g_{M}}
$$

and dropping the hats, we finally arrive at the following standard form

$$
\left\{\begin{array}{c}
\partial_{t} X_{i}=\epsilon^{2} X_{i}^{\prime \prime}-X_{i}+A M \sum_{i=1}^{N} k_{i j} X_{i} X_{j}^{n}, \quad x \in R, \\
\tau \partial_{t} M=M^{\prime \prime}+1-M-M \sum_{i, j=1}^{N} k_{i j} X_{i} X_{j}^{n}, \quad x \in R .
\end{array}\right.
$$


We shall study (1.3) on the real line $R$ for $\epsilon>0$ small. Since existence and stability of solutions might depend on $A$ we will treat it as a parameter. We look for solutions of (1.3) which are even:

$$
\begin{gathered}
X_{i}=X_{i}(|x|) \in H^{1}(R), \quad i=1, \ldots, N, \\
1-M(x)=1-M(|x|) \in H^{1}(R) .
\end{gathered}
$$

The stationary equation corresponding to (1.3) becomes

$$
\left\{\begin{array}{c}
\epsilon^{2} X_{i}^{\prime \prime}-X_{i}+A M \sum_{j=1}^{N} k_{i j} X_{i} X_{j}^{n}=0, \quad i=1, \ldots, N, \quad \in R \\
M^{\prime \prime}+1-M-M \sum_{i=1, j}^{N} k_{i j} X_{i} X_{j}^{n}=0, \quad x \in R .
\end{array}\right.
$$

We first construct cluster solutions to (1.4). To this end, we need to introduce some assumptions and notations.

Let

$$
p=n+1>1
$$

and $w$ be the unique solution of the following problem

$$
\left\{\begin{array}{l}
w^{\prime \prime}-w+w^{p}=0, w>0 \text { in } R \\
w(0)=\max _{y \in R} w(y), w(y) \rightarrow 0 \text { as }|y| \rightarrow+\infty
\end{array}\right.
$$

Put

$$
L_{\epsilon}:=\frac{N}{2 A^{1+\frac{1}{n}} k_{0}^{\frac{1}{n}}} \epsilon \int_{R}(w(y))^{n+1} d y
$$

If $\lim _{\epsilon \rightarrow 0} L_{\epsilon}<L_{0}:=\left(\frac{1}{n+1}\right)^{\frac{1}{n}} \frac{n}{n+1}$, then the following equation has two solutions:

$$
\eta^{\frac{1}{n}}(1-\eta)=L_{\epsilon}
$$

We denote the smaller one by $\eta^{s}$, where $0<\eta^{s}<\frac{1}{n+1}$ and the larger one by $\eta^{l}$, where $1>\eta^{l}>\frac{1}{n+1}$.

We now state the existence result. In fact, this is quite easy. We search for solutions of the following type

$$
X_{i}=X_{0}, \quad i=1, \ldots, N .
$$

Substituting (1.9) into (1.4), we see that $\left(X_{0}, M\right)$ satisfies

$$
\left\{\begin{array}{c}
\epsilon^{2} X_{0}^{\prime \prime}-X_{0}+A M k_{0} X_{0}^{p}=0, x \in R \\
M^{\prime \prime}+1-M-M k_{0} N X_{0}^{p}=0, x \in R
\end{array}\right.
$$

Existence of solutions to $(1.10)$ can be shown as in the case $p=2$ : the standard Gray-Scott model. Similar to the proof of Theorem 2.1 of [34], we can obtain the following existence theorem:

TheOREM 1.1. 
Assume that

$$
\epsilon<<1
$$

and

$$
\epsilon<<L_{\epsilon}, \lim _{\epsilon \rightarrow 0} L_{\epsilon}<L_{0}
$$

Then problem (1.4) admits two solutions $\left(X_{\epsilon}^{s}, M_{\epsilon}^{s}\right)=\left(X_{\epsilon, 1}^{s}, \ldots, X_{\epsilon, N}^{s}, M_{\epsilon}^{s}\right)$ and $\left(X_{\epsilon}^{l}, M_{\epsilon}^{l}\right)=\left(X_{\epsilon, 1}^{l} \ldots, X_{\epsilon, N}^{l}, M_{\epsilon}^{l}\right)$ with the following properties:

(1) all components are even functions.

(2) $X_{\epsilon, i}^{s}=\frac{1}{\left.A M_{\epsilon}^{s}(0) k_{0}\right)^{\frac{1}{n}}}(1+o(1)) w\left(\frac{|x|}{\epsilon}\right), i=1, \ldots, N$, where $w$ is the unique solution of (1.6).

(3) $M_{\epsilon}^{s}(x) \rightarrow 1, M_{\epsilon}^{l}(x) \rightarrow 1$ for all $x \neq 0$ and $M_{\epsilon}^{s}(0), M_{\epsilon}^{l}(0)$ satisfy

$$
\begin{gathered}
M_{\epsilon}^{s}(0) \sim \eta^{s}, \quad M_{\epsilon}^{l}(0) \sim \eta^{l}, \\
0<M_{\epsilon}^{s}(0)<M_{\epsilon}^{l}(0)<1 .
\end{gathered}
$$

(4) There exist $a>0, b>0$ such that

$$
\begin{array}{cl}
1-M_{\epsilon}^{s}(x) \leq C e^{-a|x|}, & 1-M_{\epsilon}^{l}(x) \leq C e^{-a|x|} \\
X_{\epsilon, i}^{s}(x) \leq C \frac{1}{\left(A M_{\epsilon}^{s}(0) k_{0}\right)^{\frac{1}{n}}} e^{-b \frac{|x|}{\epsilon}}, \quad X_{\epsilon, i}^{l}(x) \leq C \frac{1}{\left(A M_{\epsilon}^{l}(0) k_{0}\right)^{\frac{1}{n}}} e^{-b \frac{|x|}{\epsilon}}
\end{array}
$$

Finally, if $\lim _{\epsilon \rightarrow 0} L_{\epsilon}>L_{0}$, then there are no single-cluster solutions.

We note that existence of single-pulse solution has also been studied in [10].

The main goal of this paper is to study the stability and instability of the cluster solution constructed in Theorem 1.1. To this end, we first linearize the equations (1.4) around $\left(X_{\epsilon}^{s}, M_{\epsilon}^{s}\right)$ or $\left(X_{\epsilon}^{l}, M_{\epsilon}^{l}\right)$, respectively. From now on we omit the superscripts $s$ or $l$ where this is possible without confusing the reader. The linearized operator is as follows:

$$
\mathcal{L}_{\epsilon}\left(\begin{array}{c}
\phi_{\epsilon, i} \\
\psi_{\epsilon}
\end{array}\right)=\left(\begin{array}{c}
\epsilon^{2} \phi_{\epsilon, i}^{\prime \prime}-\phi_{\epsilon, i}+A M_{\epsilon} \sum_{j=1}^{N} k_{i j}\left(\phi_{\epsilon, i} X_{\epsilon, j}^{n}+n \phi_{\epsilon, j} X_{\epsilon, i} X_{\epsilon, j}^{n-1}\right) \\
+A \psi_{\epsilon} \sum_{j=1}^{N} k_{i j} X_{\epsilon, i} X_{\epsilon, j}^{n} \\
\psi_{\epsilon}^{\prime \prime}-\psi_{\epsilon}-\psi_{\epsilon} \sum_{i, j=1}^{N} k_{i j} X_{\epsilon, i} X_{\epsilon, j}^{n} \\
-M_{\epsilon} \sum_{i, j=1}^{N} k_{i j}\left(\phi_{\epsilon, i} X_{\epsilon, j}^{n}+n \phi_{\epsilon, j} X_{\epsilon, i} X_{\epsilon, j}^{n-1}\right)
\end{array}\right)
$$

where $i=1, \ldots, N$. The eigenvalue problem becomes

$$
\mathcal{L}_{\epsilon}\left(\begin{array}{c}
\phi_{\epsilon, i} \\
\psi_{\epsilon}
\end{array}\right)=\left(\begin{array}{c}
\lambda_{\epsilon} \phi_{\epsilon, i} \\
\tau \lambda_{\epsilon} \psi_{\epsilon}
\end{array}\right), i=1, \ldots, N
$$

We consider $\mathcal{L}_{\epsilon}$ in the Sobolev space $\left(H^{2}(R)\right) \otimes H^{2}(R)$ and equip $\left(H^{2}(R)\right)^{N} \oplus$ $H^{2}(R)$ with the following norm

$$
\|(X, u)\|_{\left(H^{2}(R)\right)^{N} \oplus H^{2}(R)}^{2}=\|X(y)\|_{\left(H^{2}(R)\right)^{N}}^{2}+\|u(x)\|_{H^{2}(R)}^{2} .
$$

Certainly 0 is an eigenvalue of $\mathcal{L}_{\epsilon}$. The criterion for linearized stability of a cluster solution is that the spectrum $\sigma\left(\mathcal{L}_{\epsilon}\right)$ of $\mathcal{L}_{\epsilon}$ (except for 0 ) lies in a left half plane 
$\left\{\lambda \in \mathcal{C}: \operatorname{Re}(\lambda)<-c_{0}\right\}$ where $c_{0}>0$, and that 0 is a simple eigenvalue, where $\mathcal{C}$ denotes the set of complex numbers.

In [36], the linear case $n=1$ is studied and the following result is proved.

Theorem A: Let $n=1$. Assume that

$$
\epsilon<<1, \quad \epsilon<<L_{\epsilon}, \quad \lim _{\epsilon \rightarrow 0} L_{\epsilon}<\frac{1}{4}
$$

Let $\left(X_{\epsilon}^{s}, M_{\epsilon}^{s}\right)$ and $\left(X_{\epsilon}^{l}, X_{\epsilon}^{l}\right)$ be the solutions constructed in Theorem 1.1. Then for $\epsilon<<1$, we have the following.

(1) (stability) Assume that $N \leq 4$ and $\tau<<1$. Then $\left(X_{\epsilon}^{s}, M_{\epsilon}^{s}\right)$ is linearly stable.

(2) (Instability) Assume that $N>4$. Then $\left(X_{\epsilon}^{s}, M_{\epsilon}^{s}\right)$ is linearly unstable.

(3) (Instability) $\left(X_{\epsilon}^{l}, M_{\epsilon}^{l}\right)$ is linearly unstable.

A natural question is the following: what is the effect of $n$ on the stability of cluster solutions? What is the relation between $n$ and the critical threshold? Will large $n$ increase the critical threshold? We shall answer these questions affirmatively in this paper and prove the following theorem.

Theorem 1.2. Assume that

$$
\epsilon<<1, \quad \epsilon<<L_{\epsilon}, \quad \lim _{\epsilon \rightarrow 0} L_{\epsilon}<L_{0} .
$$

Let $\left(X_{\epsilon}^{s}, M_{\epsilon}^{s}\right)$ and $\left(X_{\epsilon}^{l}, X_{\epsilon}^{l}\right)$ be the solutions constructed in Theorem 1.1. Let

$$
N_{0}:=\left\{\begin{array}{l}
4, n \leq n_{0} \sim 3.35 \\
5, n>n_{0} \sim 3.35
\end{array}\right.
$$

where $n_{0}$ satisfies

$$
\begin{gathered}
\left(\cos \frac{2 \pi}{5}\right)\left(4\left(n_{0}+2\right) \cos \frac{2 \pi}{5}+n_{0}+4\right)^{2}\left(\left(n_{0}+2\right) \cos \frac{2 \pi}{5}+2\right) \\
-\left(n_{0}+2\right) n_{0}^{2}\left(\sin \frac{2 \pi}{5}\right)^{2}=0 .
\end{gathered}
$$

Then for $\epsilon<<1$, we have the following.

(1) (stability) Assume that $N \leq N_{0}$ and $\tau<<1$. Then $\left(X_{\epsilon}^{s}, M_{\epsilon}^{s}\right)$ is linearly stable.

(2) (Instability) Assume that $N>N_{0}$. Then $\left(X_{\epsilon}^{s}, M_{\epsilon}^{s}\right)$ is linearly unstable.

(3) (Instability) $\left(X_{\epsilon}^{l}, M_{\epsilon}^{l}\right)$ is linearly unstable.

Remarks: 1. As we see from the theorem, if $p$ increases, the critical threshold can only grow by at most 1 . This means that stability is only very marginally influenced by growing interaction strength which is a new and surprising fact as one would think that stronger interaction would improve stability.

2. As in [35], we may generalize the results in this paper to the case of a general matrix $\left(k_{i j}\right)$. We will mention the results in the last section and present some examples.

It is interesting and important to know the exact threshold also to verify the validity of our model by experiment: It can now be studied if the thresholds given by theory and the one determined by experiments are the same. Furthermore, the agreement between theoretical values and numerically calculated ones for related models 
play an important role in finding which model to choose preferably. (We refer to the works quoted at the end of the introduction for related numerical investigations, in particular to [5], where among others multi-cluster states in one space dimension have been computed numerically).

Let us conclude this introduction by mentioning some related results.

In [6] the parameter dependence of stability of clusters and spirals against parasites (i.e., rival polymers which receive catalytic support from the hypercycle but do not contribute to the catalysis of any other polymer) is studied numerically. A parasite may or may not destroy the hypercycle depending on the rate constants. In [7] clusters (for $N=5$ ) are established numerically for the elementary $N$-hypercycle system,

In [5] for a closely related reaction-diffusion model the dependence of cluster states on diffusivities is shown numerically including the cluster size, their shape, and the distance between different clusters.

The effect of faulty replication on the hypercycle has been studied by an analysis of the geometry of bifurcations around steady states and numerical computations in the framework of an ODE reaction model [1].

For a cellular automata model it was shown numerically that a spiral wave structure may be stable against parasites [3]. The chaotic dynamics for this type of model has been investigated numerically in [17], [28].

There are a number of recent results on the special case $N=1, n=1$ of our model, which is then also called Gray-Scott system [13], [14]. We would like to recall them here. In [10], by using Mel'nikov method, Doelman, Kaper and Zegeling constructed single and multiple pulse solutions for (1.1) in the one-dimensional case with $D_{M}=$ $1, D_{X}=\delta^{2}<<1$, where $X_{i}=X$. In their paper [10], it is assumed that $k_{M}=g_{M} \sim$ $\delta^{2}, g_{X} \sim \delta^{2 \alpha / 3}, k_{11}=1, L=1$, where $\alpha \in\left[0, \frac{3}{2}\right)$. In this case, they showed that $M=O\left(\delta^{\alpha}\right), X=O\left(\delta^{-\frac{\alpha}{3}}\right)$. Later the stability of single and multiple pulse solutions in 1-D are obtained in [8], [9]. (The techniques are extended to other reaction-diffusion equations in [11].) Some related results on the existence and stability of solutions to the Gray-Scott model in 1-D can be found in [26] and [29].

In $R^{2}$ and $R^{3}$, Muratov and Osipov [21] have given some formal asymptotic analysis on the construction and stability of spiky solution. In [33], the system (1.1) for $N=1$ is studied on the real axis in the shadow system case, namely, $D_{M}>>$ $1, D_{X}<<1$ and $k_{M}=g_{M}=O(1), g_{X}=O(1), k_{11}=1, L=1$. The shadow system can be reduced to a single equation. For spike solutions of single equations and other systems, we refer to [15], [16], [27], [23], [24], [25], [31], [32], and the references therein.

In the general higher dimensional case rigorous existence and stability results on the Gray-Scott system have been established in [34]. The existence of one-spike solutions is proved. Their stability is established and rests upon the derivation and analysis of a related NLEP (nonlocal eigenvalue problem).

The structure of the paper is as follows:

In Section 2, we separate the eigenvalue problem into two cases: small eigenvalues and large eigenvalues. The small eigenvalue is shown to be 0 with dimension 1 . The case of large eigenvalues is then reduced to a system of nonlocal eigenvalue problems (NLEP).

In Section 3, we analyze the system of NLEP and show that it can be reduced to two eigenvalue problem-one is local but with complex coefficients, another one is a NLEP.

In Section 4, we study the two eigenvalue problems and thus finish the proof of 
Theorem 1.3.

In Section 5, we make we drop the condition that the system is a classical hypercycle and make some remarks about the general matrix case.

Finally, in Section 6, we discuss our results.

Throughout this paper, the letter $C$ will always denote various generic constants which are independent of $\epsilon$, for $\epsilon$ sufficiently small. The notation $A \sim B$ means that $\lim _{\epsilon \rightarrow 0} \frac{A}{B}=1$ and $A=O(B)$ is defined as $|A| \leq C|B|$.

2. Reduction to a system of NLEP. Let $\left(X_{\epsilon}, M_{\epsilon}\right)$ be one of the two solutions constructed in Section 1. We now study the eigenvalue problem associated with $\left(X_{\epsilon}, M_{\epsilon}\right)$. We assume that

$$
\epsilon<<L_{\epsilon}, \lim _{\epsilon \rightarrow 0} L_{\epsilon}<L_{0} .
$$

We need to analyze the following eigenvalue problem (letting $x=\epsilon y$ )

$$
\left\{\begin{array}{l}
\Delta_{y} \phi_{\epsilon, i}-\phi_{\epsilon, i}+A M_{\epsilon} \sum_{j=1}^{N} k_{i j}\left(X_{\epsilon, j}^{n} \phi_{\epsilon, i}+n \phi_{\epsilon, j} X_{\epsilon, i} X_{\epsilon, j}^{n-1}\right) \\
+A \psi_{\epsilon} \sum_{j=1}^{N} k_{i j} X_{\epsilon, i} X_{\epsilon, j}^{n}=\lambda_{\epsilon} \phi_{\epsilon, i}, y \in R \\
\Delta_{x} \psi_{\epsilon}-\psi_{\epsilon}-\psi_{\epsilon} \sum_{i, j=1}^{N} k_{i j} X_{\epsilon, i} X_{\epsilon, j}^{n} \\
-M_{\epsilon} \sum_{i, j=1}^{N} k_{i j}\left(X_{\epsilon, j}^{n} \phi_{\epsilon, i}+n X_{\epsilon, i} X_{\epsilon, j}^{n-1} \phi_{\epsilon, j}\right)=\tau \lambda_{\epsilon} \psi_{\epsilon}, x \in R \\
\lambda_{\epsilon} \in \mathcal{C} .
\end{array}\right.
$$

We assume that $\left(\phi_{\epsilon, 1}, \ldots, \phi_{\epsilon, N}, \psi_{\epsilon}\right) \in\left(H^{2}(R)\right)^{N} \oplus H^{2}(R)$.

Since $X_{\epsilon, i}=X_{0}, n=p-1, k_{i j}=k_{0} \hat{k}_{i j}=k_{0} \delta_{i, j+1}$ modulo $N$, problem (2.1) becomes

$$
\left\{\begin{array}{l}
\Delta_{y} \phi_{\epsilon, i}-\phi_{\epsilon, i}+A k_{0} M_{\epsilon} X_{0}^{p-1} k_{0} \sum_{j=1}^{N} \hat{k}_{i j}\left(\phi_{\epsilon, i}+n \phi_{\epsilon, j}\right) \\
+A k_{0} \psi_{\epsilon} X_{0}^{p}=\lambda_{\epsilon} \phi_{\epsilon, i} \\
\Delta \psi_{\epsilon}-\psi_{\epsilon}-N k_{0} \psi_{\epsilon} X_{0}^{p} \\
-M_{\epsilon} k_{0} \sum_{i, j=1}^{N} \hat{k}_{i j}\left(\phi_{\epsilon, i}+n \phi_{\epsilon, j}\right) X_{0}^{p-1}=\tau \lambda_{\epsilon} \psi_{\epsilon}
\end{array}\right.
$$

Let us first formally derive the limiting eigenvalue problems.

Since $\left(X_{0}, M_{\epsilon}\right)$ satisfies $(1.10)$, we have

$$
X_{0}(y) \sim\left(A M_{\epsilon}(0) k_{0}\right)^{-\frac{1}{p-1}} w(y) \quad \text { in } H^{1}(R)
$$

and

$$
M_{\epsilon}^{\frac{1}{p-1}}(0)\left(1-M_{\epsilon}(0)\right) \sim L_{\epsilon}:=\frac{N k_{0}}{2\left(A k_{0}\right)^{\frac{p}{p-1}}} \epsilon \int_{R} w(y)^{p} d y
$$

The eigenvalue problem is changed into

$$
\left\{\begin{array}{l}
\Delta_{y} \phi_{\epsilon, i}-\phi_{\epsilon, i}+\sum_{j=1}^{N} \hat{k}_{i j}\left(\phi_{\epsilon, i}+(p-1) \phi_{\epsilon, j}\right) w^{p-1} \\
+A k_{0}\left(A M_{\epsilon}(0) k_{0}\right)^{-\frac{p}{p-1}} \psi_{\epsilon} w^{p} \sim \lambda_{\epsilon} \phi_{\epsilon, i} \\
\Delta \psi_{\epsilon}-\psi_{\epsilon}-N k_{0}\left(A M_{\epsilon}(0) k_{0}\right)^{-\frac{p}{p-1}} \psi_{\epsilon} w^{p} \\
-M_{\epsilon} k_{0}\left(A M_{\epsilon}(0) k_{0}\right)^{-1} \sum_{i, j=1}^{N} \hat{k}_{i j}\left(w^{p-1} \phi_{\epsilon, i}+(p-1) w^{p-1} \phi_{\epsilon, j}\right) \sim \tau \lambda_{\epsilon} \psi_{\epsilon}
\end{array}\right.
$$

From the equation for $\psi_{\epsilon}$, we formally have (setting $\beta^{2}=1+\tau \lambda$ )

$$
\psi_{\epsilon}(0)=\frac{1}{2 \beta} \int_{R} e^{-\beta|x|}\left(-k_{0} N\left(A M_{\epsilon}(0) k_{0}\right)^{-\frac{p}{p-1}} \psi_{\epsilon} w^{p}\right.
$$




$$
\begin{gathered}
\left.-M_{\epsilon} k_{0}\left(A M_{\epsilon}(0) k_{0}\right)^{-1} \sum_{i, j=1}^{N} \hat{k}_{i j}\left(w^{p-1} \phi_{\epsilon, i}+(p-1) w^{p-1} \phi_{\epsilon, j}\right)\right) \\
\sim \frac{1}{2 \beta} \epsilon\left[-\psi_{\epsilon}(0) k_{0} N\left(A M_{\epsilon}(0) k_{0}\right)^{-\frac{p}{p-1}} \int_{R} w^{p}(y) d y\right. \\
\left.-M_{\epsilon}(0) k_{0} N\left(A M_{\epsilon}(0) k_{0}\right)^{-1} \int_{R} p\left(\sum_{i=1}^{N} \phi_{\epsilon, i}\right) w^{p-1} d y\right]
\end{gathered}
$$

By (1.8), we have

$$
\begin{gathered}
\psi_{\epsilon}(0) \sim-\left(1+\frac{1}{2 \beta} k_{0} N\left(A M_{\epsilon}(0) k_{0}\right)^{-\frac{p}{p-1}} \epsilon \int_{R} w^{p}\right)^{-1} \\
\left(\frac{1}{2 \beta} M_{\epsilon}(0) k_{0} N\left(A M_{\epsilon}(0) k_{0}\right)^{-1} \epsilon\left(\int_{R} w^{p-1} p\left(\sum_{i=1}^{N} \phi_{\epsilon, i}\right)\right)\right) \\
\sim-\left(1+\frac{1-M_{\epsilon}(0)}{\beta M_{\epsilon}(0)}\right)^{-1}\left(\frac{1}{2 \beta} M_{\epsilon}(0) k_{0} N\left(A M_{\epsilon}(0) k_{0}\right)^{-1} \epsilon\left(\int_{R} w^{p-1} p\left(\sum_{i=1}^{N} \phi_{\epsilon, i}\right)\right)\right)
\end{gathered}
$$

Substituting this relation into the equation for $\phi_{i}$, we obtain the following nonlocal eigenvalue problem (NLEP):

$$
\begin{gathered}
\Delta \phi_{\epsilon, i}-\phi_{\epsilon, i}+w^{p-1} \phi_{\epsilon, i}+(p-1) \sum_{j=1}^{N} \hat{k}_{i j} \phi_{\epsilon, j} w^{p-1} \\
-\frac{p\left(1-M_{\epsilon}(0)\right)}{\beta M_{\epsilon}(0)+1-M_{\epsilon}(0)} w^{p} \frac{\int_{R} w^{p-1} \sum_{i=1}^{N} \phi_{\epsilon, i}}{N \int w^{p}} \sim \lambda_{\epsilon} \phi_{\epsilon, i} .
\end{gathered}
$$

Although we have formally obtained (2.7), however we can rigorously prove the following separation of eigenvalues.

THEOREM 2.1.

Let $\lambda_{\epsilon}$ be an eigenvalue of (2.2).

(1) Suppose that $\lambda_{\epsilon} \rightarrow 0$ as $\epsilon \rightarrow 0$. Then we have $\lambda_{\epsilon}=0$ if $\epsilon$ is small enough and

$$
\left(\phi_{\epsilon}, \psi_{\epsilon}\right) \in \operatorname{span}\left\{\left(X_{\epsilon}^{\prime}, M_{\epsilon}^{\prime}\right)\right\} \text {. }
$$

(2) Suppose that $\lambda_{\epsilon} \rightarrow \lambda_{0} \neq 0$. Then $\lambda_{0}$ is an eigenvalue of the following NLEP

$$
\begin{gathered}
\Delta \phi_{i}-\phi_{i}+w^{p-1} \phi_{i}+(p-1) \sum_{j=1}^{N} \hat{k}_{i j} \phi_{j} w^{p-1} \\
-\frac{p(1-\eta)}{\beta_{0} \eta+1-\eta} w^{p} \frac{\int_{R} w^{p-1} \sum_{i=1}^{N} \phi_{i}}{N \int w^{p}}=\lambda_{0} \phi_{i}, i=1, \ldots, N
\end{gathered}
$$


where $\eta=\lim _{\epsilon \rightarrow 0} M_{\epsilon}(0), \beta_{0}=\sqrt{1+\tau \lambda_{0}}$.

Proof.

(2) follows from asymptotic analysis.

To prove (1), we can proceed exactly as in Section 6 of [34], where stability of a single cluster state is studied. Let us denote the linear operator on the left hand side of $(2.7)$ as $\mathcal{L}$, where $\mathcal{L}:\left(H^{2}(R)\right)^{N} \rightarrow\left(L^{2}(R)\right)^{N}$. The key point is to prove the following lemma:

LEMMA 2.2. (1). Let $\phi$ be an eigenfunction of (2.7) with $\lambda_{0}=0$. Then we have

$$
\phi \in \mathcal{K}_{0}:=\operatorname{span}\left\{w^{\prime}(y) \vec{e}_{0}\right\}
$$

where $\vec{e}_{0}=(1, \ldots, 1)^{\tau}$. (This implies that $\left.\operatorname{Ker}(\mathcal{L})=\mathcal{K}_{0}.\right)$

(2). The operator $\mathcal{L}$ is an invertible operator if restricted as follows

$$
\mathcal{L}: \mathcal{K}_{0}^{\perp, 1} \rightarrow \mathcal{K}_{0}^{\perp, 2}
$$

where

$$
\begin{aligned}
& \mathcal{K}_{0}^{\perp, 1}=\left\{u \in\left(H^{2}(R)\right)^{N} \mid \int_{R} u w^{\prime}(y) \overrightarrow{e_{0}}=0\right\} \\
& \mathcal{K}_{0}^{\perp, 2}=\left\{u \in\left(L^{2}(R)\right)^{N} \mid \int_{R} u w^{\prime}(y) \overrightarrow{e_{0}}=0\right\} .
\end{aligned}
$$

The proof of Lemma 2.2 is technical and is similar to Theorem 4.1 of [36].

The rest of the proof is exactly the same as in Section 6 of [33]. For the sake of limited space, we omit the details here.

3. Analysis of system of NLEP. In this section we analyze the nonlinear eigenvalue problem (NLEP) which we have obtained in Section 2. To this end, we introduce two eigenvalue problems: the first is the following eigenvalue problem with complex coefficients

$$
\left\{\begin{array}{c}
\Delta \phi-\phi+w^{p-1} \phi+(p-1) \sigma w^{p-1} \phi=\lambda \phi \\
\sigma=\sigma_{R}+\sqrt{-1} \sigma_{I}=e^{i \theta}, \theta \in(-\pi, \pi], \quad \phi \in H^{1}(R),
\end{array}\right.
$$

where $w$ is defined by (1.6).

The second is a nonlocal eigenvalue problem (NLEP):

$$
\Delta \phi-\phi+p w^{p-1} \phi-\frac{p(1-\eta)}{\eta \sqrt{1+\tau \lambda}+1-\eta} \frac{\int_{R} w^{p-1} \phi}{\int_{R} w^{p}} w^{p}=\lambda \phi, \phi \in H^{2}(R)
$$

where

$$
0<\eta<1, \tau \geq 0, \lambda \in \mathcal{C}, \lambda=\lambda_{R}+i \lambda_{I}, \lambda_{R} \geq 0
$$

and we take principal branch for $\sqrt{1+\tau \lambda}$.

We show that the study of NLEP (2.7) can be reduced to the study of (3.1) and (3.2). We say an eigenvalue problem is stable if there exists a constant $c_{0}>0$ such that all eigenvalues $\lambda$ we have $\operatorname{Re}(\lambda)<-c_{0}$. We say it is unstable if there exists an eigenvalue $\lambda$ with $\operatorname{Re}(\lambda)>0$. 
We then have the following

LEMMA 3.1. Suppose that (3.2) with $\eta=\lim _{\epsilon \rightarrow 0} M_{\epsilon}(0)$ has no Hopf bifurcation (as given in Lemma 4.6 below). Then (2.7) is stable if and only if both (3.1) (with $\sigma=e^{-\frac{2 j \pi \sqrt{-1}}{N}}, j=1, \ldots, N-1$ ) and (3.2) (with $\eta=\lim _{\epsilon \rightarrow 0} M_{\epsilon}(0)$ ) are stable.

Proof.

(1) Suppose (3.1) and (3.2) are stable. We now show that (2.7) is stable, too: Asuming that there exists $\lambda_{0} \geq 0$ such that (2.7) holds we show that

$$
\phi_{i}=0, \quad i=1, \ldots, N \text {. }
$$

We first take care of the nonlocal terms. Adding all equations for $i=1, \ldots, N$, we get

$$
\begin{gathered}
\Delta\left(\sum_{i=1}^{N} \phi_{i}\right)-\left(\sum_{i=1}^{N} \phi_{i}\right)+p w^{p-1}\left(\sum_{i=1}^{N} \phi_{i}\right) \\
-p \frac{1-\eta}{\beta \eta+1-\eta} \frac{\int_{R}\left(\sum_{i=1}^{N} \phi_{i}\right) w^{p-1}}{\int_{R} w^{p}} w^{p}=\lambda_{0} \phi_{i} .
\end{gathered}
$$

Since (3.2) is stable and we have no Hopf bifurcation, we have

$$
\sum_{i=1}^{N} \phi_{i}=0
$$

Suppose (3.3) holds so the nonlocal terms in (NLEP) all vanish. We end up with the following:

$$
\Delta \phi_{i}-\phi_{i}+w^{p-1} \phi_{i}+(p-1) \sum_{j=1}^{N} \hat{k}_{i j} \phi_{j} w^{p-1}=\lambda_{0} \phi_{i}
$$

After diagonalizing $\hat{k}_{i j}$ (keeping the notation for $\phi_{i}$ ) we get

$$
\Delta \phi_{i}-\phi_{i}+\left(1+(p-1) e^{2 \pi i \sqrt{-1} / N}\right) w^{p-1} \phi_{i}=\lambda_{0} \phi_{i}
$$

Since (3.1) is stable, we have $\phi_{i}=0$. Therefore (2.7) is stable.

(2) Suppose (3.2) is unstable. Then there exists an eigenfunction $\phi_{0} \neq 0$ with an eigenvalue $\lambda_{0}$ to (3.2) such that $\operatorname{Re}\left(\lambda_{0}\right)>0$. Now we take $\phi_{1}=\ldots=\phi_{N}=\phi_{0}$ in (2.7) and we see that (2.7) also admits the eigenvalue $\lambda_{0}$. So (2.7) is unstable.

On the other hand, suppose (3.2) is not unstable and (3.1) is not stable. Since (3.2) has no Hopf bifurcations, (3.2) is stable. Then similar to (1), we must have

$$
\sum_{i=1}^{N} \phi_{i}=0
$$

and so all the nonlocal terms vanish. We are left with the following local eigenvalue problem

$$
\Delta \phi_{i}-\phi_{i}+w^{p-1} \phi_{i}+(p-1) \sum_{j=1}^{N} \hat{k}_{i j} w^{p-1} \phi_{j}=\lambda \phi_{i}, \sum_{i=1}^{N} \phi_{i}=0
$$

It is easy to see that (3.6) is not stable because (3.1) is not stable. Lemma 3.1 is thus proved. 
4. Study of the two eigenvalue problems. In this section, we study two eigenvalue problems (3.1) and (3.2) derived from Section 3. The analysis presented in this section is the key estimate for this paper.

To study (3.1) and (3.2), we first collect some important properties associated with the function $w$. We first study some local eigenvalue problems.

LEMMA 4.1. (1) The linear operator

$$
\left\{\begin{array}{c}
L_{0} \phi:=\phi^{\prime \prime}-\phi+p w^{p-1} \phi \\
\phi \in H^{1}(R)
\end{array}\right.
$$

has the kernel

$$
\operatorname{Ker}\left(L_{0}\right)=\operatorname{span}\left\{w^{\prime}(y)\right\}
$$

(2) The eigenvalue problem (EVP)

$$
(E V P) \quad\left\{\begin{array}{c}
\phi^{\prime \prime}-\phi+\mu w^{p-1} \phi=0 \\
\phi \in H^{1}(R)
\end{array}\right.
$$

admits the following set of eigenvalues

$$
\begin{aligned}
& \mu_{1}=1, v_{1}=\operatorname{span}\{w\} \\
& \mu_{2}=p, v_{2}=\operatorname{Ker}\left(L_{0}\right),
\end{aligned}
$$

$$
\mu_{3}>p
$$

(3) If $\mu_{R}>0$, then the following eigenvalue problem

$$
\left\{\begin{array}{c}
\phi^{\prime \prime}-\phi+w^{p-1} \phi+\mu_{R} w^{p-1} \phi=\lambda \phi \\
\mu_{R}>0, \phi \in H^{1}(R)
\end{array}\right.
$$

admits a positive (principal) eigenvalue $\lambda_{1}$ such that

$$
-\lambda_{1}=\inf _{\phi \in H^{1}(R) \backslash\{0\}} \frac{\int_{R}\left(\phi^{\prime}\right)^{2}+\phi^{2}-\left(1+\mu_{R}\right) w^{p-1} \phi^{2}}{\int_{R} \phi^{2}}<0 .
$$

(4) Let $\phi$ (complex-valued) satisfy the following eigenvalue problem

$$
\left\{\begin{array}{c}
\phi^{\prime \prime}-\phi+w^{p-1} \phi+(p-1) \sigma w^{p-1} \phi=\lambda \phi \\
\operatorname{Re}(\sigma) \leq 0, \quad \phi \in H^{1}(R), \quad \lambda \neq 0
\end{array}\right.
$$

Then

$$
\operatorname{Re}(\lambda) \leq-c_{0}<0
$$

Proof. The proof is similar to Lemma 3.1 of [36]. We omit the details.

We are ready to study the first eigenvalue problem (3.1). We consider $\theta$ as a parameter. By Lemma 4.1 (3) and a perturbation argument, for $|\theta|$ small, there is 
an unstable eigenvalue $\lambda$ for problem (3.1), i.e. $\lambda=\lambda_{R}+i \lambda_{I}$ where $\lambda_{R}>0$. On the other hand, by Lemma 4.1 (4), for $|\theta| \geq \frac{\pi}{2}$, problem (3.1) has only stable eigenvalues, i.e. $\lambda=\lambda_{R}+i \lambda_{I}$ where $\lambda_{R}<0$. Now if we vary $\theta$, then there must be a point $\theta^{h}$ such that for $\theta=\theta^{h}$, problem (3.1) has a Hopf bifurcation, i.e. there is an eigenvalue $\lambda=i \lambda_{I}$. Let us now compute $\theta^{h}$. That is

LEMMA 4.2. Let $\phi$ (complex-valued) satisfy the eigenvalue problem (3.1). Then there exists some $\theta^{h}$ with $\theta^{h}=\arccos \sigma_{R}$, where $\sigma_{R}$ is the unique zero with $0<\sigma_{R}<1$ of the following polynomial

(4.1) $\left.g(\sigma):=\sigma_{R}\left(4(p+1) \sigma_{R}+p+3\right)^{2}(p+1) \sigma_{R}+2\right)-(p+1)(p-1)^{2}\left(1-\sigma_{R}^{2}\right)$

such that (1) If

$$
|\theta|>\theta^{h}
$$

then

$$
\operatorname{Re}(\lambda) \leq-c_{0}<0
$$

(2) If

$$
|\theta|<\theta^{h}
$$

then there exists an eigenvalue $\lambda$ with $\operatorname{Re}(\lambda)>0$.

(3) If $|\theta|=\theta^{h}$, then there exists an eigenvalue $\lambda$ with $\lambda=i \lambda_{I}$.

Proof. We are looking for a Hopf bifurction for problem (3.1). Therefore we have to solve

$$
\Delta \phi-\phi+(1+(p-1) \sigma) w^{p-1} \phi=\lambda \phi
$$

with

$$
\lambda=\sqrt{-1} \lambda_{I}
$$

(i.e. the real part $\lambda_{R}$ of $\lambda$ vanishes) and

$$
\sigma=\sigma_{R}+\sqrt{-1} \sigma_{I}, \quad|\sigma|^{2}=\sigma_{R}^{2}+\sigma_{I}^{2}=1
$$

Let

$$
\gamma=\sqrt{1+\lambda}, \mu=1+(p-1) \sigma, \phi=w^{\gamma} F
$$

Then $F$ satisfies

$$
F^{\prime \prime}+2 \gamma \frac{w^{\prime}}{w} F^{\prime}+\left(\mu-\left(\gamma+\frac{2}{p+1} \gamma(\gamma-1)\right)\right) w^{p-1} F=0 .
$$

Next we introduce the following new variable

$$
z=\frac{1}{2}\left(1-\frac{w^{\prime}}{w}\right)
$$

Then

$$
\frac{w^{\prime}}{w}=1-2 z, w^{p-1}=2(p+1) z(1-z), \frac{d z}{d x}=(p-1) z(1-z) .
$$


This yields the following equation for $F$ as function of $z$

$$
z(1-z) F^{\prime \prime}+(c-(a+b+1) z) F^{\prime}-a b F=0
$$

where

(4.6) $a+b+1=2+\frac{4 \gamma}{p-1}, a b=2 \frac{2 \gamma(\gamma-1)-(\mu-\gamma)(p+1)}{(p-1)^{2}}, c=1+\frac{2 \gamma}{p-1}$.

The solutions to (4.5) are standard hypergeometric functions. Now there are two solutions to (4.5):

$$
F(a, b ; c ; z), z^{1-c} F(a-c+1, b-c+1 ; 2-c ; z) .
$$

Since by our construction, $F$ is regular at $z=0$. At $z=1, F(a, b ; c ; z)$ has a singularity

$$
\lim _{z \rightarrow 1}(1-z)^{-(c-a-b)} F(a, b ; c ; z)=\frac{\Gamma(c) \Gamma(a+b-c)}{\Gamma(a) \Gamma(b)}
$$

where $c-a-b=-\frac{2 \gamma}{p-1}$. Note that since $\lambda=\sqrt{1+i \lambda_{I}}$, the real part of $\gamma$ is posivive. So a solution that is regular at both $z=0$ and $z=1$ can only exist if $\Gamma(x)$ has a pole at $a$ or $b$, respectively. In other words, $a=0,-1,-2, \ldots$ or $b=0,-1,-2, \ldots$.

From (4.6), we compute that

$$
a=\frac{2 \gamma}{p-1}-\alpha
$$

or

$$
b=\frac{2 \gamma}{p-1}-\alpha
$$

where $\alpha$ satisfies

$$
\alpha^{2}+\alpha-\frac{2(p+1)}{(p-1)^{2}} \mu=0 .
$$

By symmetry we may assume that $a=\frac{2 \gamma}{p-1}-\alpha=-l, l \geq 0$ and $\alpha=\alpha_{R}+\sqrt{-1} \alpha_{I}$. So we to solve the system

$$
\left\{\begin{array}{c}
\alpha_{R}^{2}+\alpha_{R}-\alpha_{I}^{2}-\frac{2(p+1)}{(p-1)^{2}}\left(1+(p-1) \sigma_{R}\right)=0 \\
\frac{2 \gamma}{p-1}=\alpha-l
\end{array}\right.
$$

Since we take the principal branch for $\gamma=\sqrt{1+i \lambda_{I}}$, it follows that

$$
\alpha>l \text {. }
$$

Moreover we have

$$
\frac{4}{(p-1)^{2}}=\left(\alpha_{R}-l\right)^{2}-\alpha_{I}^{2}
$$

which implies that

$$
\alpha_{R} \geq l+\frac{2}{p-1}
$$


On the other hand, we have

$$
\begin{aligned}
& \frac{4}{(p-1)^{2}}=\left(\alpha_{R}-l\right)^{2}-\alpha_{I}^{2}=\alpha_{R}^{2}-\alpha_{I}^{2}-2 l \alpha_{R}+l^{2} \\
& =-(2 l+1) \alpha_{R}+l^{2}+\frac{2(p+1)}{(p-1)^{2}}\left(1+(p-1) \sigma_{R}\right) .
\end{aligned}
$$

So we obtain

$$
\alpha_{R}=\frac{1}{2 l+1}\left(l^{2}+\frac{2}{p-1}+\frac{2(p+1)}{p-1} \sigma_{R}\right) .
$$

By (4.9), we have

$$
\frac{1}{2 l+1}\left(l^{2}+\frac{2}{p-1}+\frac{2(p+1)}{p-1} \sigma_{R}\right) \geq l+\frac{2}{p-1}
$$

which is impossible unless $l=0$ or $l=1$. For $l=1$ we just recover the case $l=0$ with the eigenfunction $w^{\prime}$ given by Lemma 4.1 (1). This clearly does not correspond to Hopf bifurcation.

For Hopf bifurcation to occur we must have $a=0$ or $b=0$. In this case, we have

$$
\alpha_{R}=\frac{2}{p-1}+\frac{2(p+1)}{p-1} \sigma_{R}, \alpha_{I}=\frac{2(p+1)}{(p-1)\left(2 \alpha_{R}+1\right)} \sigma_{I} .
$$

Substituting this relation into (4.8) we obtain that $\sigma_{R}$ must be a zero of the polynomial $g$ defined by (4.1).

In summary, Hopf bifurcation can occur only at the point $\sigma_{R}^{h}$ such that $g\left(\sigma_{R}^{h}\right)=0$. Since such a point is unique, we conclude that for $|\theta|<\theta^{h}=\arccos \sigma_{R}^{h}$, there is unstable eigenvalues and for $|\theta|>\theta^{h}$, all eigenvalues are stable. $\square$

Let us now analyze the polynomial $g\left(\sigma_{R}\right)$ for $\sigma=e^{\frac{2 \pi \sqrt{-1}}{N}}$. We note that as $p \rightarrow+\infty$, the zeroes of $g$ approach the zeroes of

$$
g_{0}\left(\sigma_{R}\right)=\sigma_{R}^{2}\left(4 \sigma_{R}+1\right)^{2}-\left(1-\sigma_{R}^{2}\right)
$$

The zero of $g_{0}$ is approximately 0.3726 . Thus as $p$ becomes large, $N_{\text {critical }}$-the critical threshold- can not exceed 6. In fact, one can compute explicitly that in the case $p>n_{0}+1$, where $n_{0}$ is given by $(1.20)$, we have $g\left(\cos \left(\frac{2 \pi}{N}\right)\right)<0$ for $N \leq 5$ and $g\left(\cos \left(\frac{2 \pi}{N}\right)\right)>0$ for $N \geq 6$. In the case $p<n_{0}+1$, we have $g\left(\cos \left(\frac{2 \pi}{N}\right)<0\right.$ for $N \leq 4$ and $g\left(\cos \left(\frac{2 \pi}{N}\right)\right)>0$ for $N \geq 5$. That is we have the following corollary

Corollary 4.3. For $N \leq N_{0}$, the problem (3.1) is stable, while for $N>N_{0}$, problem (3.1) is unstable, where

$$
N_{0}= \begin{cases}4 & \text { if } p \leq p_{0} \\ 5 & \text { if } p \geq p_{0}\end{cases}
$$

and $p_{0} \sim 4.35$.

We next study the NLEP (3.2). We first recall the following lemma 
LEMma 4.4. [37] Consider the following eigenvalue problem

$$
\Delta \phi-\phi+p w^{p-1} \phi-\gamma(p-1) \frac{\int_{R} w^{p-1} \phi}{\int_{R} w^{p}} w^{2}=\lambda \phi, \phi \in H^{2}(R) .
$$

Then if $\gamma \leq p$, we have $\operatorname{Re}(\lambda)<-c_{1}<0$ for some $c_{1}>0$.

From Lemma (4.4), it follows immediately we have the following

LEMMA 4.5. Consider the eigenvalue problem (3.2).

(1) If $\tau<<1$ and $0<\eta<\frac{1}{p}$. Let $\lambda_{0} \neq 0$ be an eigenvalue of (3.2). Then we have $\operatorname{Re}\left(\lambda_{0}\right) \leq-c_{1}$ for some $c_{1}>0$.

(2) Suppose that $\frac{1}{p}<\eta<1$, then problem (3.2) admits a real eigenvalue $\lambda_{0}$ with $\lambda_{0} \geq c_{2}>0$ for some $c_{2}>0$.

Proof.

(1). When $\tau=0$, we have

$$
\frac{p(1-\eta)}{\eta \sqrt{1+\tau \lambda}+1-\eta}=p(1-\eta)>p-1
$$

if $0<\eta<\frac{1}{p}$. By Lemma 4.4, we must have that $\lambda_{R}<-c_{1}<0$. The case $\tau<<1$ follows from a perturbation argument.

(2). Assume that $\frac{1}{p}<\eta<1$. By Lemma 4.1 (3), $L_{0}$ has a positive eigenvalue $\mu_{1}>0$. Consider the following function

$$
h(\alpha)=\int_{R}\left(\left(L_{0}-\alpha\right)^{-1} w^{p-1}\right) w^{p-1} .
$$

It is easy to see that

$$
h^{\prime}(\alpha)=\int_{R}\left(\left(L_{0}-\alpha\right)^{-2} w^{p-1}\right) w^{p-1}=\int_{R}\left[\left(L_{0}-\alpha\right)^{-1} w^{p-1}\right]^{2}>0
$$

and

$$
\lim _{\alpha \rightarrow \mu_{1}} h(\alpha)=+\infty
$$

Next we consider the function

$$
\rho(\lambda)=\frac{\eta \sqrt{1+\tau \lambda}+1-\eta}{p(1-\eta)}-1-\left(\int_{R} w^{p}\right)^{-1} \lambda h(\lambda) .
$$

Note that

$$
\rho(0)=\frac{1}{p(1-\eta)}-1>0
$$

since $\frac{1}{p}<\eta<1$. On the other hand,

$$
\lim _{\lambda \rightarrow \mu_{1}-} \rho(\lambda)=-\infty .
$$

Hence there must exist an $\lambda_{0} \in\left(0, \mu_{1}\right)$ such that $\rho\left(\lambda_{0}\right)=0$.

It is easy to see that this $\lambda_{0}>0$ is an eigenvalue of (3.2). 
In the general case $\tau>0,0<\eta<\frac{1}{p}$, there are no analytic results for problem (3.2). Fortunately, we can use hypergeometric functions and generalized hypergeometric functions to reduce problem (3.2) to a computable problem. Such an idea has already been used in [8]. However, here we propose a different transformation so that the eigenvalue problem becomes computable more easily. We recall that by Lemma 4.3 (2) for $\tau=0$, all eigenvalues are stable. So if we vary $\tau$, either we obtain stability or Hopf bifurcation. All we need is to compute when Hopf bifurcation occurs.

Let us first introduce the so-called generalized Gauss function. Let $a_{1}, a_{2}, \ldots, a_{A}$ and $b_{1}, b_{2}, \ldots, b_{B}$ be two sequences of numbers. Consider the following series

$$
\begin{aligned}
& 1+\frac{a_{1} a_{2} \ldots a_{A}}{b_{1} b_{2} \ldots b_{B}} \frac{z}{1 !}+\frac{\left(a_{1}+1\right)\left(a_{2}+1\right) \ldots\left(a_{A}+1\right)}{\left(b_{1}+1\right)\left(b_{2}+1\right) \ldots\left(b_{B}+1\right)} \frac{z^{2}}{2 !}+\ldots \\
& \equiv{ }_{A} F_{B}\left\{\begin{array}{ccccc}
a_{1}, & a_{2}, & \ldots, & a_{A} & ; \\
& & & & z \\
b_{1}, & b_{2}, & \ldots, & b_{B} & ;
\end{array}\right\}
\end{aligned}
$$

${ }_{A} F_{B}$ is called generalized Gauss function or generalized hypergeometric function.

Now we have the following lemma. The proof is similar to Lemma 3.4 of [36]. So we omit the details.

LEMma 4.6. Let $\lambda=\sqrt{-1} \lambda_{I}$ be an eigenvalue of problem (3.2). Then $\lambda$ is a solution of the following algebraic equation

$$
\begin{aligned}
& \frac{(3 p-1)\left(p^{2}-1-\lambda\right)}{p\left(p^{2}-1\right)} \frac{\eta \sqrt{1+\tau \lambda}+1-\eta}{p(1-\eta)}
\end{aligned}
$$

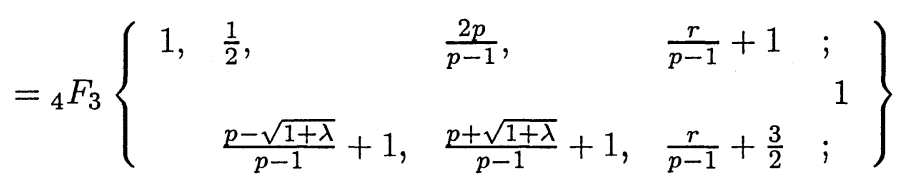

By Lemma (4.6), problem (3.2) can be solved by using Mathematica. We will not produce any numerical results here. The readers can refer to [8] for some numerical results.

5. General Matrix Case. Theorems 1.1 and 1.3 can be extended to more general matrices $\left(k_{i j}\right)$.

Let us consider system (1.3):

$$
\left\{\begin{aligned}
\partial_{t} X_{i} & =\epsilon^{2} X_{i}^{\prime \prime}-X_{i}+A M \sum_{i=1}^{N} k_{i j} X_{i} X_{j}^{n}, \quad x \in R \\
\tau \partial_{t} M & =M^{\prime \prime}+1-M-M \sum_{i, j=}^{N} k_{i j} X_{i} X_{j}^{n}, \quad x \in R
\end{aligned}\right.
$$

where $\left(k_{i j}\right)$ is a general matrix. To ensure existence, we put the following symmetric condition

$$
\sum_{i=1}^{N} k_{i j}=\sum_{j=1}^{N} k_{i j}=k_{0}
$$


Then Theorem 1.1 holds true without any change.

The main problem is the stability. To this end, we need to put an extra assumption

$(\mathrm{H} 1) \quad[1+\operatorname{spec}(\mathcal{B})] \cap \operatorname{spec}(\mathrm{EVP})=\{p\}$,

where $\mathcal{B}=\left(\hat{k}_{i j}\right)$ and the EVP is defined in Lemma 4.1.

The following is our main result on stability.

TheOREM 5.1. Assume that

$$
\epsilon<<1, \quad \epsilon<<L_{\epsilon}, \quad \lim _{\epsilon \rightarrow 0} L_{\epsilon}<L_{0}
$$

and that assumption (H1) holds. Let $\left(X_{\epsilon}^{s}, M_{\epsilon}^{s}\right)$ and $\left(X_{\epsilon}^{l}, X_{\epsilon}^{l}\right)$ be the solutions given in Theorem 1.1.

Let $\sigma=\sigma_{R}+\sqrt{-1} \sigma_{I}$ be an eigenvalue of $\left(\hat{k}_{i j}\right)$ and let the polynomial $g$ be defined as in (4.1).

Then for $\epsilon<<1$, we have the following.

(1) (stability) Suppose that $\tau<<1$. Assume that $\sigma=1$ is a simple eigenvalue and that for all $\sigma$ with $\sigma_{R}>0$, we have $g(\sigma)<0$. Then $\left(X_{\epsilon}^{s}, M_{\epsilon}^{s}\right)$ is linearly stable.

(2) (Instability) Assume that either $\sigma=1$ is not simple or there exists $\sigma \neq 1$ with $\sigma_{R}>0$ such that $g(\sigma)>0$. Then $\left(X_{\epsilon}^{s}, M_{\epsilon}^{s}\right)$ is linearly unstable.

(3) (Instability) $\left(X_{\epsilon}^{l}, M_{\epsilon}^{l}\right)$ is linearly unstable.

The proof of Theorem 5.1 is the same as that of Theorem 1.2. We omit the details. Note that the analysis in Sections $2-4$ deals with general matrices $\hat{k}_{i j}$ and is not restricted to merely the hypercycle case $\hat{k}_{i j}=\delta_{i, j+1}$ modulo $N$. Let us now apply Theorem 5.1 to some interesting examples.

Our first example is the following cyclical bi-diagonal matrix

$$
\left(k_{i j}\right)=k_{0}\left(\begin{array}{ccccc}
1-\alpha & \alpha & 0 & \cdots & 0 \\
0 & 1-\alpha & \alpha & \ldots & 0 \\
0 & 0 & 1-\alpha & \ldots & 0 \\
\cdots & \ldots & \ldots & \cdots & \alpha \\
\alpha & 0 & \cdots & 0 & 1-\alpha
\end{array}\right)_{N \times N}, \quad k_{0}>0 .
$$

It is easy to calculate that the eigenvalues are $\sigma=1-\alpha\left(1-e^{2 \pi j \sqrt{-1} / N}\right), j=1, \ldots, N$ and are all simple.

We substitute $\sigma$ into the polynomial and compute the critical threshold $N_{\text {critical }}$. It turns out that $N_{\text {critical }}$ depends on both $\alpha$ and $p: N_{\text {critical }}$ will increase of the order $\alpha$ as $\alpha$ increases but $N_{\text {critical }}$ increases only slowly in $p$. In fact, let us fix $\alpha$ and consider the case $p \rightarrow+\infty$. Then as $p \rightarrow+\infty$, the zeroes of $f$ approach the zeroes of the polynomial

$$
g_{0}(\sigma):=\sigma_{R}^{2}\left(4 \sigma_{R}+1\right)^{2}-\sigma_{I}^{2}
$$

which was defined in (4.10) above. Substituting $\sigma_{R}=1-\alpha+\alpha \cos (\theta), \sigma_{I}=\alpha \sin (\theta)$ into (4.10), we obtain the following

$$
\rho(\theta, \alpha)=(1-\alpha+\alpha \cos (\theta))^{2}(5-4 \alpha+4 \cos (\theta))^{2}-\alpha^{2}\left(1-\cos ^{2}(\theta)\right)=0 .
$$


Note that for $\alpha$ large and $\alpha \theta=O(1)$

$$
\theta \sim \frac{5}{\alpha}
$$

which by $\theta=2 \pi / N_{0}$ implies that

$$
N_{0} \sim \frac{2 \pi}{5} \alpha
$$

On the other hand for $p=2$ we get under the same assumptions

$$
N_{0} \sim \frac{2 \pi \sqrt{3}}{17 \sqrt{5}} .
$$

In both cases the critical threshold $N_{0}$ grows linearly in alpha but it is bigger by the factor $\frac{17}{\sqrt{15}} \sim 4.39$ in the first case. Thus in this case large $p$ changes quantitative but not the qualitative behavior of $N_{0}$. This shows a more striking change of behavior than in the hypercycle case.

Our second example is a system with $(N-1)$ interactions.

$$
\left(k_{i j}\right)=k_{0}\left(\begin{array}{ccccc}
0 & 1 & 1 & \ldots & 1 \\
1 & 0 & 1 & \ldots & 1 \\
1 & 1 & 0 & \ldots & 1 \\
\ldots & \ldots & \ldots & \ldots & 1 \\
1 & 1 & \ldots & 1 & 0
\end{array}\right)_{N \times N}, \quad k_{0}>0
$$

The eigenvalues of $\left(\hat{k}_{i j}\right)$ are $\sigma=1$ (which is simple) and $\sigma=0$. The small cluster state is stable for all $N$ independent of the rate $p$.

From all the previous examples, we see as a general trend that if the system is not too much dominated by diagonal terms we have stability. Otherwise, a parasite emerges. This means that cooperative behavior in contrast with self-enhancement is needed to stabilize the cluster.

For large $p$ stability in increased somewhat. We point to the second example where the stability threshold $N_{0}$ for large $\alpha$ grows linearly in $\alpha$ and large $p$ can improve $N_{0}$ by a constant of about 4.39. In the case $\alpha>1$ (which means that the diagonal becomes negative and the off-diagonal elements are positive and bigger than the diagonal), this describes self-inhibition coupled with cooperative enhancement and leads to particularly good stability.

Furthermore, the second and the third example indicate that coupling between more and more different components $X_{i}$ also improves stability. Note that in the last example the system can be arbitrarily large.

6. Discussion. We have studied a general system of $N+1$ equations with nonlinear rate $n$ describing the interaction of $N$ polymer species which catalyse each other in a hypercyclic way and are all composed of the same type of monomer. In the special case $N=1, n=1$ the system reduces to the well-known Gray-Scott system.

We study the case of single-cluster solutions in the whole 1-D space. These are in some sense the simplest concentrated solutions in 1-D. This case appears to be relevant if the early biochemical reactions take place in very thin lines for example on the edges of rocks. 
Besides the existence proof we provide the first rigorous results on stability for cluster states of a hypercyclic system with nonlinear rate. Namely, we establish the exact threshold between stability and instability in terms of the system size and the nonlinear rate $n$. It is shown that as $n \leq 3.35$, the stability regimes extends exactly in the range $N=1,2,3,4$. If $n>3.35$, the stability regime extends to $N=1,2,3,4,5$. This shows that the maximum critical threshold for hypercycle system with nonlinear rate is 5 . This result might be important for making predictions about the outcome of experiments in biological applications and also for the testing of the validity of the models used. Furthermore, now a comparison of this theoretical result with numerical computations becomes possible.

We have also studied reaction-diffusion systems with nonlinear rate and general connection matrix $\left(k_{i j}\right)$. There we have observed that the nonlinear rate can help increase the critical threshold $N_{\text {critical }}$ when there is large self-inhibition (Example 1, Section 5). We show that large self-inhibition and to some lesser extent large $n$ can help stabilize large reaction system.

So how can very large systems be stabilized?

One possibility is to increase self-inhibition and the nonlinear rate, as in Example 1 , Section 5.

Another possibility which is frequently observed in nature is by the formation of a block-diagonal structure. Then, since the spectra of different blocks are independent, they can for example be chosen as small hypercycles which are stable up to size 5 . On the other hand, by assembling a large number of blocks the system can become arbitrarily large and still be stable.

In fact, it is much simpler to create systems which display block-diagonal structure with only a few interactions than systems with many components catalysing each other. Therefore block-diagonal structures are frequently observed and are very important for natural phenomena.

Finally, let us recall attention to the point made in the introduction numerically it is known that parasites may destroy stable cluster states. Our results complement the picture by the rigorously proved fact that even pure cluster states may turn unstable if they become two large. This implies that the hypercycle although it has some very preferable properties (see the beginning of the introduction) on the other hand it has an inherent instability behavior which may be an obstruction to the evolution of large biological systems.

Acknowledgments: This research is supported by an Earmarked Research Grant from RGC of Hong Kong. MW thanks the Department of Mathematics at The Chinese University of Hong Kong for their kind hospitality.

\section{REFERENCES}

[1] M. A. Andrade, J. C. Nuño, F. Morán, F. Montero, and G. J. Mpitsos, Complex dynamics of a catalytic network having faulty replication into error-species, Physica D, 63 (1993), pp. 21-40.

[2] R. A. Barrio, C. Varea, J. L. Aragòn, P. K. Maini, A two-dimensional numerical study of spatial pattern formation in interacting Turing systems, Bull. Math. Biol., 61 (1999), pp. 483-505.

[3] M.C. BoerliJST AND P. Hogeweg, Spiral wave structure in pre-biotic evolution: hypercycles stable against parasites, Physica D, 48 (1991), pp. 17-28.

[4] K.-S. CHENG AND W.-M. NI, On the structure of the conformal Gaussian curvature equation on R, Duke Math. J., 62 (1991), pp. 721-737. 
[5] P. Chacón And J.C. NUÑo, Spatial dynamics of a model for prebiotic evolution, Physica D, 81 (1995), pp. 398-410.

[6] M. B. CRONHJORT AND C. Blomberg, Hypercycles versus parasites in a two-dimensional partial differential equations model, Journal Theoret. Biol., 169 (1994), pp. 31-49.

[7] M. B. CRONHJORT AND C. BlomBerg, Cluster compartmentalization may provide resistance to parasites for catalytic networks, Physica D, 101 (1997), pp. 289-298.

[8] A. Doelman, A. Gardner, AND T.J. Kaper, Stability analysis of singular patterns in the 1-D Gray-Scott model: A matched asymptotic approach, Physica D, 122 (1998), pp. 1-36.

[9] A. Doelman, A. Gardner, And T.J. Kaper, A stability index analysis of 1-D patterns of the Gray-Scott model, Methods Appl. Anal., 7 (2000).

[10] A. Doelman, T. Kaper, AND P. A. Zegeling, Pattern formation in the one-dimensional Gray-Scott model, Nonlinearity, 10 (1997), pp. 523-563.

[11] A. Doelman, R. GARDner, AND T. J. KAPER, Large stable pulse solutions in reactiondiffusion equations, Indiana Univ. Math. J., 49, No. 4 (2000).

[12] M. EIGEN AND P. SCHUSTER, The hypercycle: A principle of natural selforganisation,(Springer, Berlin, 1979).

[13] P. GRAY AND S.K. SCOTT, Autocatalytic reactions in the isothermal, continuous stirred tank reactor: isolas and other forms of multistability, Chem. Eng. Sci., 38 (1983), pp. 29-43.

[14] P. Gray AND S.K. ScotT, Autocatalytic reactions in the isothermal, continuous stirred tank reactor: oscillations and instabilities in the system $A+2 B \rightarrow 3 B, B \rightarrow C$, Chem. Eng. Sci., 39 (1984), pp. 1087-1097.

[15] C. GUI AND J. WEI, Multiple interior peak solutions for some singularly perturbed Neumann problems, J. Differential Equations, 158 (1999), pp. 1-27.

[16] C. GUI, J. Wei AND M. WINTER, Multiple boundary peak solutions for some singularly perturbed Neumann problems, Ann. Inst. H. Poincaré Anal. Non Linéaire, 17 (2000), pp. 249-289.

[17] K. Lindgren AND M.G. Nordahl, Evolutionary dynamics of spatial games, Physica D, 75 (1994), pp. 292-309.

[18] M.J. Metcalf, J. H. Merkin And Scott, S. K, Oscillating wave fronts in isothermal chemical systems with arbitrary powers of autocatalysis, Proc. Roy. Soc. London Ser. A, 447 (1994), pp. 155-174.

[19] P. M. MCCABE, J. A. LeACH AND D. J. NeEdham, The evolution of travelling waves in fractional order autocatalysis with decay, I. Permanent form travelling waves, SIAM J. Appl. Math., 59 (1999), no. 3, pp. 870-899.

[20] P. M. MCCABE, J.A. LEACH AND D. J. NeEdham, The evolution of travelling waves in fractional order autocatalysis with decay, II. The initial boundary value problem, SIAM J. Appl. Math., 60 (2000), no. 5, pp. 1707-1748.

[21] C.B. Muratov, V.V. Osipov, Spike autosolitions in Gray-Scott model, Los Alamoseprint, patt-sol/9804001, submitted.

[22] D.J. NEEDHAM AND J.H. MERKIN, The development of travelling waves in a simple isothermal chemical system with general orders of autocatalysis and decay, Philos. Trans. Roy. Soc. London Ser. A, 337 (1991), pp. 261-274.

[23] W.-M. NI AND I. TAKAGI, On the shape of least energy solution to a semilinear Neumann problem, Comm. Pure Appl. Math., 41 (1991), pp. 819-851.

[24] W.-M. Ni AND I. TAKAGI, Locating the peaks of least energy solutions to a semilinear Neumann problem, Duke Math. J., 70 (1993), pp. 247-281.

[25] W.-M. Ni, I. TAKagi AND E. YAnAgidA, Tohoku Math. J., to appear.

[26] Y. NishiURA AND D. UEYAMA, A skeleton structure of self-replicating dynamics, Physcia D., 130 (1999), pp. 73-104.

[27] W.-M. NI, Diffusion, cross-diffusion, and their spike-layer steady states, Notices Amer. Math. Soc., 45 (1998), pp. 9-18.

[28] M. A. NoWAK AND R. M. MAY, Evolutionary games and spatial chaos, Nature, 359 (1993), pp. 826-829.

[29] J. Reynolds, J. Pearson and S. Ponce-Dawson, Dynamics of self-replicating spots in reaction-diffusion systems, Phy. Rev. E, 56 (1) (1997), pp. 185-198.

[30] J. Reynolds, J. Pearson and S. Ponce-Dawson, Dynamics of self-replicating patterns in reaction diffusion systems, Phy. Rev. Lett., 72 (1994), pp. 2797-2800.

[31] J. WEI, Uniqueness and eigenvalue estimates of boundary spike solutions, Proc. Royal Soc. Edinburgh, Section A (Mathematics), to appear.

[32] J. WEI, On single interior spike solutions of Gierer-Meinhardt system: uniqueness, spec- 
trum estimates and stability analysis, Euro. J. Appl. Math., 10 (1999), pp. 353-378.

[33] J. WEI, Existence, stability and metastability of point condensation patterns generated by Gray-Scott system, Nonlinearity, 12 (1999), pp. 593-616.

[34] J. WEI, On two dimensional Gray-Scott model: existence of single pulse solutions and their stability, Phys. D, 148(2001), pp. 1-2, 20-48.

[35] J. WEI AND M. WINTER, On a two dimensional reaction-diffusion system with hypercyclical structure, Nonlinearity, 13 (2000), pp. 2005-2032.

[36] J. WeI AND M. WINTER, Critical Threshold and Stability of Cluster Solutions for Large Reaction-Diffusion Systems in $R^{1}$, SIAM J. Math. Anal., to appear.

[37] J. WEI AND M. Winter, A note on a nonlcal eigenvalue problem, submitted. 
J. WEI AND M. WINTER 